\title{
Withholding and withdrawing life support: difficult decisions around care at the end of life
}

\author{
Kevin J. Solverson, MD, MSc, FRCPC • Amanda L. Roze des Ordons, MD, MMEd, FRCPC • \\ Christopher J. Doig, MD, MSc, FRCPC
}

Received: 7 September 2017/Accepted: 17 October 2017/Published online: 30 November 2017

(C) Canadian Anesthesiologists' Society 2017

In this issue of the Journal, the Canadian Critical Care Society (CCCS) released a position paper by Bandrauk et al. ${ }^{1}$ on withholding and withdrawing life-sustaining treatment (WWLST). The authors' objective was to create a framework for healthcare professionals to help them understand and develop an approach to end-of-life care decision-making in the intensive care unit (ICU). End-oflife care and WWLST are common issues in the ICU, and as the authors have identified in their article, these practices require careful attention to a number of ethical factors and legal precedents as well as to the context of the patient/family and healthcare professionals. This editorial provides additional comments on WWLST and end-of-life care.

When a critically ill patient is unlikely to survive or the medical therapies and prognosis are no longer aligned with the patient's previously expressed goals, it is appropriate for healthcare professionals to consider withdrawing LST and, if appropriate, to transition their focus to palliative

K. J. Solverson, MD, MSc, FRCPC .

A. L. Roze des Ordons, MD, MMEd, FRCPC .

C. J. Doig, MD, MSc, FRCPC ( $\square)$

Department of Critical Care Medicine, Cumming School of Medicine, University of Calgary, 3134 Hospital Drive NW, Calgary, AB T2N 5A1, Canada

e-mail: cdoig@ucalgary.ca

A. L. Roze des Ordons, MD, MMEd, FRCPC

Department of Anesthesia, Cumming School of Medicine,

University of Calgary, Calgary, AB, Canada

A. L. Roze des Ordons, MD, MMEd, FRCPC

Department of Oncology (Palliative Care), Cumming School of

Medicine, University of Calgary, Calgary, AB, Canada

C. J. Doig, MD, MSc, FRCPC

Department of Community Health Sciences, Cumming School of

Medicine, University of Calgary, Calgary, AB, Canada care. These should always be difficult decisions. For most patients, the transition to end-of-life care occurs without conflict, and understanding and agreement between the patient, family, and healthcare team facilitate a caring and dignified death. Although rare, when consensual agreement is not achieved, it may result in significant distress for all involved. Poor experiences with the healthcare system, both current and past, including complications of treatment or lack of respect for beliefs or concerns, may predictably lead to conflict. The patient and family may feel alienated and resentful towards the medical team as they advocate for treatments that have been determined to be medically unhelpful and inappropriate. Even less frequently, but often with significant media attention, some disputes over discontinuing LST are brought before the judicial system for resolution, adding to the stress and suffering of all involved.

While there are times when conflict may be unavoidable, an emphasis on prevention is critical. As Bandrauk et al. have stressed, sensitive communication with patients and their families about WWLST facilitates consensual decision-making. The words on the Canadian Medical Association's Coat of Arms, i.e., integrity and compassion - "integritate et misericordia" - are foundational in these situations. Integrity and a healthy dose of humility can remind healthcare professionals of their position of authority and the vulnerability of their patients and their families. The nuances of language and how information is presented can easily sway decisions. Compassion is essential in recognizing and acknowledging the distress a family experiences as they participate in decisions about whether the life of a loved one may either continue in an uncertain state or end in loss. Before entering into conversations about prognosis and recommendations for treatment, it is important to take the 
time to gain an empathetic understanding of the patient's life and their family relationships. This approach can help convey these principles while building the trust needed for decision-making and support. Learning about patient and family cultural and spiritual beliefs allows a better understanding of the perspectives, decisions, and reactions to conversations about WWLST. As spoken by Osler, "Care more for the individual patient than for the special features of the disease." These words of wisdom remain apropos for discussions with patients and their families around end-of-life care in the ICU.

The postgraduate education of physicians should include learning how to approach end-of-life conversations. Research in this area has identified that postgraduate trainees place a high priority on end-of-life communication skills. ${ }^{2}$ There are often multifaceted personal, interprofessional, and system issues (e.g., resource constraints, policies around documentation, and disconnects within the healthcare team) that contribute to missed opportunities for effecting improvement in these difficult conversations. ${ }^{3}$ Considering the frequency of challenging discussions in the ICU longitudinal communication programs-particularly those with multidimensional and interprofessional components-is an important element of critical care training. Our group has described a longitudinal curriculum that includes role play with simulated family members and group debriefing as well as feedback from healthcare professionals and families following family meetings in the clinical setting. ${ }^{4}$ This type of learning should not be limited to trainees. We encourage all healthcare providers engaged in critical care to participate in interprofessional continuing education that considers contextual issues such as spirituality, culture, and personal values.

The decriminalization of medical assistance in dying (MAID) in Canada has changed and will continue to change the landscape of care at the end of life, with implications for perspectives around WWLST. In Canada, MAID differs somewhat from other jurisdictions, for example, it permits both patient self-administration of physician-prescribed medications to cause death as well as healthcare provider direct administration of medications to cause death. This has created moral conflict for some providers and faith-based care facilities. A recent roundtable meeting of ethicists and critical care physicians reported equipoise on a number of ethical issues around MAID. ${ }^{5}$ Some participants considered the moral agency of the physician in WWLST and MAID to be equivalent, as both procedures respect patient autonomy, relieve suffering, and lead to the same outcome. While the goal of both procedures is to relieve suffering, the means through which this is achieved differs. For WWLST, suffering is alleviated by discontinuing medical interventions that are causing more harm than benefit (e.g., mechanical ventilation, invasive intravascular lines, catheters, etc.), and death is neither intended nor required, although usually foreseeable. Conversely, the intent of MAID is to cause death as a means to relieve suffering. Strong individual opinions among patients, families, and healthcare professionals may be as varied as those of the aforementioned experts, impacting the possibility of achieving consensual end-of-life decisions at the bedside.

In supporting the moral and legal distinction between WWLST and MAID, the CCCS position paper has important implications. Patients and their families who are not in favour of MAID and do not understand how it differs from WWLST may be resistant to discontinuing LST when medically appropriate. They may fear or accuse healthcare professionals and hospitals of a primary intent to hasten death, not understanding that recommendations to limit or withdraw interventions are based on the absence of treatment benefit. Other contextual features, such as demand for ICU services and bed capacity, may accentuate mistrust. While intentionally hastening the dying process is illegal except for the very specific context of MAID, attending physicians ${ }^{6}$ and nurses in some countries have reported this practice. ${ }^{7}$ The Belgian Society of Intensive Care Medicine recently released a statement paper in support of using medications in the absence of patient discomfort to "improve the quality of dying" by shortening the dying process. ${ }^{8}$ The CCCS position paper will open a conversation about current WWLST practices in Canadian ICUs and how the recommendations could be applied in practice to facilitate transparent end-of-life care that respects patient values and preferences while acknowledging the experiences of those caring for them.

As advances in medicine and surgery allow patients with serious medical conditions to live longer than was historically possible, conversations around end-of-life care and decisions about WWLST will become increasingly important. Situated within an evolving clinical framework, the ethical issues surrounding WWLST will require continual reappraisal. From a very personal perspective, a loved one's death is a very intimate part of a relationship. As healthcare professionals, we are privileged to be invited into this relationship. This privilege carries significant responsibility. Integrity, compassion, and a principle-based interprofessional approach to the challenges encountered in conversations about WWLST will enable care, both leading up to and at the end of life, to be individualized to the dynamic and contextual needs of our patients and their families. 


\section{L'abstention et}

\section{l'interruption des}

traitements de maintien de

la vie : des décisions

difficiles concernant les soins

de fin de vie

Dans ce numéro du Journal, la Société canadienne de soins intensifs (SCSI) publie un exposé de principe de Bandrauk et coll. ${ }^{1}$ portant sur l'abstention et l'interruption des traitements de maintien de la vie. L'objectif des auteurs était de créer un cadre destiné aux professionnels de la santé afin de les aider à comprendre et mettre au point une approche à la prise de décision concernant les soins de vie à l'unité de soins intensifs (USI). Les soins de fin de vie et l'abstention et l'interruption des traitements de maintien de la vie sont des questions récurrentes à l'USI et, comme les auteurs le mentionnent dans leur article, ces pratiques exigent de porter une attention toute particulière à plusieurs questions éthiques et précédents jurisprudentiels ainsi qu'au contexte particulier du patient, de sa famille et des professionnels de la santé concernés. Cet éditorial propose quelques réflexions supplémentaires concernant l'abstention et l'interruption des traitements de maintien de la vie et les soins de fin de vie.

Lorsqu'un patient en état critique a peu de chances de survie ou que les traitements médicaux et le pronostic ne sont plus en accord avec les volontés précédemment exprimées par le patient, il est alors de mise que les professionnels de la santé envisagent d'interrompre les traitements de maintien de la vie et, si la situation le justifie, se concentrent plutôt sur des soins palliatifs. Ces décisions seront toujours difficiles. Pour la plupart des patients, la transition vers des soins de fin de vie se passe sans conflit, et la compréhension et le consensus entre le patient, sa famille et l'équipe de soins de santé facilitent une mort humaine et dans la dignité. Bien que de telles situations soient rares, lorsqu'on ne parvient pas à une décision consensuelle, une détresse considérable peut alors affecter toutes les personnes concernées. Les mauvaises expériences avec le système de santé, qu'elles soient actuelles ou passées, comme par exemple les complications suite à un traitement ou le manque de respect face aux croyances et aux préoccupations des patients, peuvent, comme on s'en doute, mener à la discorde. Le patient et sa famille pourraient se sentir aliénés et en vouloir à l'équipe médicale alors qu'ils plaident pour l'administration de traitements qui sont peu utiles du point de vue médical ou inappropriés. Plus rarement encore, mais provoquant souvent l'attention soutenue des médias, certaines disputes concernant l'interruption du traitement de survie sont portées devant les tribunaux afin d'être résolues, ajoutant encore au stress et aux souffrances de toutes les personnes concernées.

Bien que parfois le conflit soit inévitable, il est absolument essentiel de prioriser une approche de prévention. Comme le soulignent Bandrauk et coll., une communication toute en sensibilité avec les patients et leurs familles concernant l'abstention et l'interruption des traitements de maintien de la vie facilite une prise de décision consensuelle. Les mots ornant le blason de l'Association médicale canadienne, soit «Intégrité et compassion» (integritate et misericordia) sont fondamentaux dans ces situations. L'intégrité, accompagnée d'une bonne dose d'humilité, permettra aux professionnels de la santé de se rappeler de leur position d'autorité et de la vulnérabilité de leurs patients et de leurs familles. Les nuances langagières et la façon de présenter les informations peuvent facilement influencer les décisions. La compassion est essentielle pour reconnaître et prendre conscience de la détresse ressentie par une famille alors qu'elle doit décider si la vie d'une personne aimée devrait se poursuivre dans un état incertain ou s'achever. Avant d'amorcer toute discussion concernant le pronostic et les recommandations de traitement, il est important de prendre le temps de comprendre, avec compassion, la vie du patient et es relations familiales qui l'entourent. Une telle approche peut favoriser la transmission de ces principes tout en créant la confiance nécessaire à la prise de décision et au soutien. En se familiarisant avec les convictions culturelles et spirituelles d'un patient et de sa famille, le professionnel de la santé est plus à même de comprendre les perspectives, les décisions et les réactions concernant l'abstention et l'interruption des traitements de maintien de la vie. Comme le disait Osler, "Intéressez-vous davantage au patient qu'aux caractéristiques particulières de sa maladie. »Ces mots sages demeurent bien à propos quand il s'agit de discuter des soins de fin de vie à l'USI avec nos patients et leurs familles.

La formation postuniversitaire des médecins devrait intégrer des stratégies visant à apprendre comment aborder les conversations concernant la fin de vie. Les recherches dans ce domaine ont découvert que les résidents en spécialité accordent une priorité élevée aux compétences en communication concernant la fin de vie. ${ }^{2}$ Bien souvent, des questions personnelles, interprofessionnelles et systémiques plurifactorielles (qu'il s'agisse de contraintes au niveau des ressources, des politiques concernant la documentation, ou encore d'un sentiment de déconnexion avec l'équipe de soins) gâchent les occasions d'apporter 
des améliorations à ces conversations difficiles. ${ }^{3}$ Étant donné la fréquence des discussions difficiles à l'USI, des programmes longitudinaux de communication à l'USI particulièrement ceux présentant des composantes multidimensionnelles et interprofessionnelles - constituent donc un élément important de la formation en soins intensifs. Notre groupe a décrit un programme de cours longitudinal qui intègre des jeux de rôle avec des membres de la famille simulés et un débriefing de groupe ainsi que des rétroactions des professionnels de la santé et des familles suite à des réunions de famille dans un contexte clinique. ${ }^{4}$ Ce type de formation ne devrait pas être offert uniquement aux résidents. Nous encourageons tous les intervenants impliqués dans les soins de santé à l'USI à participer à des formations continues interprofessionnelles qui traitent de questions contextuelles telles que la spiritualité, la culture et les valeurs personnelles.

La décriminalisation de l'aide à mourir au Canada a modifié et continuera de modifier le paysage des soins de fin de vie, en raison de ses implications sur les perspectives entourant l'abstention et l'interruption des traitements de maintien de la vie. Au Canada, l'aide à mourir est quelque peu différente de celle proposée dans d'autres pays. Par exemple, elle permet l'auto-administration, par le patient, de médicaments prescrits par le médecin afin de provoquer la mort, mais permet également l'administration directe, par un professionnel de la santé, de médicaments causant la mort. Cette double facette a entraîné des conflits moraux pour certains médecins et établissements de soins de santé à vocation religieuse. Récemment, une table ronde réunissant éthiciens et intensivistes a rapporté qu'ils étaient parvenus à un équilibre concernant plusieurs questions éthiques entourant l'aide à mourir. ${ }^{5}$ Pour certains participants, le libre-arbitre du médecin est tout autant mis en jeu dans l'abstention et l'interruption des traitements de maintien de la vie que dans l'aide à mourir, car les deux interventions respectent l'autonomie du patient, soulagent les souffrances, et ont le même résultat. Bien que l'objectif de l'une et l'autre intervention soit de soulager les souffrances, les moyens pour y parvenir sont différents. Dans le cas de l'abstention et de l'interruption des traitements de maintien de la vie, les souffrances sont allégées en cessant toute intervention médicale qui cause davantage de mal que de bienfaits (par ex., la ventilation mécanique, les lignes intravasculaires invasives, les cathéters, etc.), et la mort n'est ni voulue ni nécessaire, bien qu'en général prévisible. À l'inverse, l'intention de l'aide à mourir est de provoquer la mort en tant que moyen de soulager les souffrances. Des opinions très tranchées parmi les patients, les familles et les professionnels de la santé peuvent varier tout autant que parmi les experts mentionnés ci-dessus, ce qui peut avoir un impact sur la possibilité de prendre des décisions consensuelles concernant les soins de fin de vie au chevet du patient.

En exposant la distinction morale et légale entre l'abstention et l'interruption des traitements de maintien de la vie et l'aide à mourir, l'exposé de principe de la SCSI a d'importantes implications. Les patients et familles qui ne sont pas en faveur de l'aide à mourir et ne comprennent pas en quoi cette approche diffère de l'abstention et l'interruption des traitements de maintien de la vie pourraient être réfractaires à l'idée d'interrompre un traitement de maintien de la vie lorsque cela est médicalement adapté. Ils pourraient avoir peur ou accuser les professionnels de la santé et les hôpitaux d'avoir pour but premier d'accélérer la mort, ne comprenant pas que les recommandations visant à limiter ou interrompre certaines interventions se fondent sur l'absence de bienfaits du traitement. D'autres paramètres contextuels, tels que la demande pour les services de l'USI et le nombre de lits, pourraient accentuer le manque de confiance. Alors que l'accélération intentionnelle du processus de mourir est illégale sauf dans le contexte très spécifique de l'aide à mourir, dans certains pays, des médecins ${ }^{6}$ et infirmières ont rapporté cette pratique. ${ }^{7}$ La Société belge de soins intensifs et de médecine d'urgence (SIZ) a récemment publié une déclaration soutenant l'utilisation de médicaments en l'absence d'inconfort du patient afin «d'améliorer la qualité de la mort », raccourcissant ainsi le processus de décès. ${ }^{8}$ L'exposé de principe de la SCSI servira de fondement pour lancer la conversation concernant les pratiques actuelles d'abstention et d'interruption des traitements de maintien de la vie dans les USI canadiennes et la façon dont les recommandations pourront être appliquées dans la pratique afin de faciliter des soins de fin de vie transparents respectant les valeurs et préférences des patients tout en tenant compte des expériences des personnes prenant soin d'eux.

Alors que les progrès en médecine et en chirurgie permettent aux patients atteints de maladie grave de vivre plus longtemps que jamais, les conversations portant sur les soins de fin de vie et les décisions concernant l'abstention et l'interruption des traitements de maintien de la vie vont devenir de plus en plus fréquentes. Dans le contexte d'un cadre clinique en évolution, les questions éthiques entourant l'abstention et l'interruption des traitements de maintien de la vie devront constamment être réévaluées. D'un point de vue très personnel, la mort d'un proche fait partie de l'intimité d'une relation. En tant que professionnels de la santé, nous avons le privilège d'être invités dans cette relation. Ce privilège s'accompagne d'une lourde responsabilité. L'intégrité, la compassion, et une approche interprofessionnelle fondée sur les principes face aux défis que comportent les conversations concernant l'abstention et l'interruption des traitements de maintien de 
la vie permettront que les soins, qu'il s'agisse de ceux jusqu'à ou entourant la fin de vie, soient personnalisés selon les besoins dynamiques et contextuels de nos patients et de leurs familles.

Conflicts of interest The authors report no conflicts of interest.

Editorial responsibility This submission was handled by Dr. Hilary P. Grocott, Editor-in-Chief, Canadian Journal of Anesthesia.

Financial support None.

Conflit d'intérêt Les auteurs ne déclarent aucun conflit d'intérêt.

Responsabilité éditoriale Cet article a été traité par Dr Hilary P. Grocott, rédacteur en chef, Journal canadien d'anesthésie.

Soutien financier Aucun.

\section{References}

1. Bandrauk N, Downar J, Paunovic B; Canadian Critical Care Society Ethics Committee. Withholding and withdrawing of life- sustaining treatment: The Canadian Critical Care Society position paper. Can J Anesth 2018; 65. DOI:10.1007/s12630-017-1002-1.

2. Roze des Ordons A, Ajjawi R, Macdonald J, Sarti A, Lockyer J, Hartwick M. Palliative and end of life care communication as emerging priorities in postgraduate medical education. Can Med Educ J 2016; 7: e4-21.

3. Roze des Ordons AL, Lockyer J, Hartwick M, Sarti A, Ajjawi R. An exploration of contextual dimensions impacting goals of care conversations in postgraduate medical education. BMC Palliat Care 2016; 15: 34.

4. Roze des Ordons AL, Doig CJ, Couillard P, Lord J. From communication skills to skillful communication: a longitudinal integrated curriculum for critical care medicine fellows. Acad Med 2017; 92: 501-5.

5. Goligher EC, Ely EW, Sulmasy DP, et al. Physician-assisted suicide and euthanasia in the ICU: a dialogue on core ethical issues. Crit Care Med 2017; 45: 149-55.

6. Sprung CL, Ledoux D, Bulow HH, et al. Relieving suffering or intentionally hastening death: where do you draw the line? Crit Care Med 2008; 36: 8-13.

7. Asch DA. The role of critical care nurses in euthanasia and assisted suicide. N Engl J Med 1996; 334: 1374-9.

8. Vincent $J L$, Schetz $M$, De Waele JJ, et al. "Piece" of mind: end of life in the intensive care unit statement of the Belgian Society of Intensive Care Medicine. J Crit Care 2014; 29: 174-5. 